\title{
A segurança do paciente no centro cirúrgico: papel da equipe de enfermagem
}

\author{
The patient safety at the surgical center: \\ role of the nursing team
}

\author{
Bárbara Ribeiro ${ }^{1}$, Janaina Samantha Martins de Souza ${ }^{2}$
}

\section{Resumo}

Objetivo: identificar o papel da equipe de enfermagem de um centro cirúrgico quanto à aplicação da segurança do paciente. Material e Método: trata-se de um estudo descritivo de caráter exploratório com abordagem quantitativa, realizado com profissionais da equipe de enfermagem atuantes no centro cirúrgico de uma instituição hospitalar privada, localizada na Serra Gaúcha, no estado do Rio Grande do Sul, Brasil. Os dados foram coletados através de questionários formulados por 25 perguntas, que posteriormente foram armazenados em planilhas no Microsoft ${ }^{\circledR}$ Windows $^{\circledR}$ Excel $^{\circledR} 2010$ em forma de tabelas para análise estatística descritiva. Resultados: considerando os critérios de inclusão e exclusão, a amostra foi composta por 24 questionários válidos. Os dados analisados evidenciaram predomínio de concordância entre os enfermeiros e os técnicos em enfermagem referente à adesão da segurança do paciente em centro cirúrgico. Conclusão: a pesquisa ressaltou as dificuldades que os profissionais relatam durante sua jornada de trabalho. Tornou-se evidente a insatisfação dos mesmos em relação ao quantitativo dos profissionais de enfermagem, que, de acordo com eles, é um número insuficiente em relação à demanda das necessidades de um processo de cuidar e de segurança do paciente.

Palavras-chave: Pacientes; Enfermeiro; Cirurgia segura.

\begin{abstract}
Objective: to identify the role of the nursing team in a surgical center regarding the application of patient safety. Material and Method: this is an exploratory descriptive study with a quantitative approach, carried out with professionals of the nursing team of the surgical center of a private institution, located in the Serra Gaúcha, in Rio Grande do Sul state, Brazil. Data were collected through questionnaires formulated by 25 questions, which were later stored in Microsoft ${ }^{\circledR}$ Windows ${ }^{\circledR}$ Excel $^{\circledR} 2010$ spreadsheets in the form of tables for descriptive statistical analysis.
\end{abstract}

\footnotetext{
${ }^{1}$ Graduanda em Enfermagem no Centro Universitário da Serra Gaúcha (FSG), Caxias do Sul, Rio Grande do Sul, Brasil. E-mail: barbara-ribb_@hotmail.com

${ }^{2}$ Doutorado em Medicina e Ciências da Saúde pela Pontifícia Universidade Católica do Rio Grande do Sul (PUCRS), Porto Alegre, Rio Grande do Sul, Brasil. Professora na Faculdade Fátima, Caxias do Sul, Rio Grande do Sul, Brasil. Professora no Centro Universitário da Serra Gaúcha, Caxias do Sul, Rio Grande do Sul, Brasil.
} 
Results: considering the inclusion and exclusion criteria, the sample consisted of 24 valid questionnaires. The analyzed data showed a high level of agreement between nurses and nursing technicians regarding adherence to patient safety in the operating room. Conclusion: the research highlighted the difficulties that professionals report during their workday. It became evident their dissatisfaction in relation to the number of nursing professionals, which, according to them, is an insufficient number in relation to the demand for the needs of a patient care and safety process.

Keywords: Patients; Nurse; Safe surgery.

\section{Introdução}

Os avanços tecnológicos e científicos na área da saúde têm propiciado o aumento no número de intervenções cirúrgicas, das quais, muitas são realizadas em condições consideradas inseguras, interferindo assim na promoção e na recuperação da saúde dos pacientes. A ocorrência de eventos adversos acentua-se nas instituições hospitalares, representando grave problema de saúde pública. ${ }^{(1)}$

Na última década a preocupação com a segurança do paciente tornou-se constante para todo o setor de saúde em todas as suas dimensões, sendo o foco principal ambientes de alto risco para a ocorrência de eventos adversos, como é o caso do centro cirúrgico. ${ }^{(2)}$

Os eventos adversos no centro cirúrgico ocorrem devido à complexidade dos procedimentos, falhas nos equipamentos de anestesia, falta de pessoal capacitado, equipe cirúrgica trabalhando sob pressão, uso de novas tecnologias com pouco conhecimento, entre outros fatores. ${ }^{(1)}$ Apesar de as intervenções cirúrgicas integrarem a assistência à saúde, contribuindo para a prevenção de agravos à integridade física e à perda de vidas, ainda respondem por grande proporção das mortes e danos temporários ou permanentes, provocados pelo processo assistencial, considerados evitáveis. ${ }^{(3)}$

Dessa forma, os centros cirúrgicos são considerados cenários complexos, suscetíveis a erros, que podem gerar complicações aos pacientes e até levá-los à morte. Em países desenvolvidos o índice de complicações importantes em procedimentos cirúrgicos é de $3 \%$ a $16 \%$, e a taxa de mortalidade é de $0,4 \%$ a $0,8 \%$. Já em países em desenvolvimento, estimam-se taxas de mortalidade de $5 \%$ a $10 \%$ em cirurgias de grande porte. Aproximadamente, metade desses eventos, ou complicações, pode ser considerada evitável. ${ }^{(4)}$

A segurança compõe uma importante dimensão da qualidade, definindo-se como o direito dos indivíduos de terem os riscos de um dano desnecessário associado com o cuidado de saúde reduzidos a um mínimo aceitável. Esta, por sua vez, é um dos maiores desafios para a excelência da qualidade no serviço de saúde. ${ }^{(5)}$

Nesta perspectiva, busca-se com a segurança do paciente a redução e a diminuição de atos considerados inseguros, atrelados ao sistema de assistência à saúde, com o emprego de melhores práticas, no intuito de evitar a ocorrência de eventos adversos. ${ }^{(2)}$

No ambiente cirúrgico, o enfermeiro tem um papel fundamental em garantir que melhores práticas de cuidado proporcionem a segurança do paciente. $\mathrm{Na}$ busca pela qualidade dos cuidados em saúde, este profissional tem o potencial para elaborar processos de melhoria contínua da assistência, a partir do planejamento de estratégias para diminuição de erros e boas práticas assistenciais, contando sempre com os integrantes da sua equipe de enfermagem. Isso é resultado da proximidade do enfermeiro e da equipe com o paciente, pois estes profissionais estão presentes em todas as etapas do período perioperatório. ${ }^{(3)}$

Este estudo teve como objetivo identificar o papel da equipe de enfermagem de um centro cirúrgico quanto à aplicação da segurança do paciente. 


\section{Material e Método}

Trata-se de um estudo descritivo de caráter exploratório com abordagem quantitativa. O mesmo foi realizado com profissionais da equipe de enfermagem atuantes no centro cirúrgico de uma instituição hospitalar privada de médio porte, localizada na região da Serra Gaúcha, no estado do Rio Grande do Sul, Brasil, no período de setembro a outubro de 2020 .

O centro cirúrgico da instituição engloba o bloco cirúrgico, a sala de recuperação e o centro de materiais e esterilizações (CME). O bloco cirúrgico é composto por sete salas equipadas para a realização de procedimentos de alta complexidade, como neurocirurgias e cirurgias cardíacas e traumatológicas. São realizados cerca de 1.300 procedimentos cirúrgicos mensais de pequeno, médio e grande porte no bloco.

A coleta dos dados foi realizada através de um questionário contendo 25 perguntas que abordaram o perfil profissional da equipe de enfermagem, a avaliação da equipe quanto ao momento de chegada, permanência e saída do paciente da sala cirúrgica e referente à instituição e à unidade do centro cirúrgico. As perguntas foram elaboradas com base no Protocolo para Cirurgia Segura, do Ministério da Saúde (MS), ${ }^{(6)}$ e na tese de doutorado intitulada "A cultura de segurança do paciente: validação de um instrumento de mensuração para o contexto hospitalar brasileiro". (7)

Devido à pandemia de Covid-19, não foi possível que as autoras/pesquisadoras adentrassem nas dependências do centro cirúrgico da instituição para a coleta de dados. Sendo assim, o questionário e o Termo de Consentimento Livre e Esclarecido (TCLE) foram entregues em envelopes identificados, na recepção do centro cirúrgico, pelas autoras. Estes permaneceram lá pelo período de sete dias. Após os profissionais da equipe de enfermagem responderem os questionários, a coordenadora do centro cirúrgico os recolheu e os colocou novamente na recepção, sinalizando para que as autoras os pegassem de volta.
Para participar da pesquisa foram adotados os critérios de inclusão: estar há mais de três meses atuando no centro cirúrgico da instituição e aceitar participar voluntariamente do estudo, assinando o TCLE. Já os critérios de exclusão foram: estar de férias, atestado ou licença no momento da coleta de dados, responder de forma incompleta o questionário e negar participar voluntariamente do estudo ou não assinar o TCLE.

Ao todo foram recolhidos 29 questionários, mas 5 estavam respondidos de forma incompleta; desse modo, considerando os critérios de inclusão e exclusão, a amostra foi composta por 24 questionários válidos.

Os dados obtidos nos questionários foram armazenados em planilhas do software Microsoft ${ }^{\circledR}$ Windows ${ }^{\circledR}$ Excel $^{\circledR} 2010$ em forma de tabelas para análise estatística descritiva.

A equipe de enfermagem que atua no centro cirúrgico é composta, de forma geral, pelo enfermeiro e pelo técnico ou auxiliar de enfermagem. ${ }^{(8)}$

$\mathrm{O}$ enfermeiro e o técnico de enfermagem participam desde a preparação de materiais que serão utilizados, até a atuação direta em campo operatório e, ainda, na sala de recuperação pós-anestésica. ${ }^{(13)}$

A pesquisa foi realizada de acordo com os aspectos éticos, tendo início somente após aprovação do Comitê de Ética em Pesquisa (CEP) no dia 17 de outubro de 2020 e mediante o Certificado de Apresentação para Apreciação Ética (CAAE) $n^{\circ}$ 36883420.0.0000.5523 e Parecer $n^{\circ}$ 4.273.530.

\section{Resultados}

Dos 24 profissionais da equipe de enfermagem que responderam o questionário, 22 (91,7\%) são do sexo feminino. Relativo à idade, $11(45,8 \%)$ possuem média de 31 a 40 anos. Quanto à escolaridade, $20(83,4 \%)$ são técnicos de enfermagem e 4 $(16,6 \%)$ enfermeiros. Em relação ao tempo de formação profissional, 11 (29,2\%) possuem média de 11 anos a 15 anos. E referente ao tempo de atuação dos profissionais em centro cirúrgico, 12 (50\%) atuam em média de 6 anos a 10 anos (Tabela 1). 
Tabela 1 - Perfil profissional da equipe de enfermagem da unidade de centro cirúrgico de uma instituição hospitalar privada da Serra Gaúcha - 2020.

\begin{tabular}{|c|c|c|c|}
\hline Variáveis & Características & $\mathbf{N}$ & $\%$ \\
\hline \multirow[t]{2}{*}{ Gênero } & Masculino & 2 & $8,3 \%$ \\
\hline & Feminino & 22 & $91,7 \%$ \\
\hline \multirow[t]{5}{*}{ Faixa de idade } & De 20 a 30 anos & 3 & $12,5 \%$ \\
\hline & De 31 a 40 anos & 11 & $45,8 \%$ \\
\hline & De 41 a 50 anos & 7 & $29,2 \%$ \\
\hline & Acima de 50 anos & 1 & $4,2 \%$ \\
\hline & Não informaram & 2 & $8,3 \%$ \\
\hline \multirow[t]{2}{*}{ Formação profissional } & Enfermeiro (a) & 4 & $16,6 \%$ \\
\hline & Técnico (a) de enfermagem & 20 & $83,4 \%$ \\
\hline \multirow[t]{5}{*}{ Tempo de formação } & De 1 ano a 5 anos & 3 & $12,5 \%$ \\
\hline & De 6 anos a 10 anos & 8 & $33,4 \%$ \\
\hline & De 11 anos a 15 anos & 7 & $29,2 \%$ \\
\hline & De 16 anos a 20 anos & 4 & $16,6 \%$ \\
\hline & Não informaram & 2 & $8,3 \%$ \\
\hline \multirow[t]{5}{*}{ Tempo de atuação em centro cirúrgico } & De 1 ano a 5 anos & 7 & $29,2 \%$ \\
\hline & De 6 anos a 10 anos & 12 & $50 \%$ \\
\hline & De 11 anos a 15 anos & 2 & $8,3 \%$ \\
\hline & De 16 anos a 20 anos & 2 & $8,3 \%$ \\
\hline & De 20 anos a 30 anos & 1 & $4,2 \%$ \\
\hline
\end{tabular}

Fonte: dados da pesquisa.

Na sequência, na Tabela 2 foi possível ob- Além disso, pôde-se notar que os 24 profissionais servar que $23(95,8 \%)$ dos profissionais realizam (100\%) verificam os antecedentes alérgicos dos a checagem de realização de jejum do paciente. pacientes.

Tabela 2 - Avaliação da equipe de enfermagem da unidade de centro cirúrgico de uma instituição hospitalar privada da Serra Gaúcha, referente à chegada do paciente ao hospital até sua entrada na sala cirúrgica. 2020.

\begin{tabular}{lccc}
\hline Variáveis & Alternativas & $\mathbf{N}$ & $\mathbf{\%}$ \\
\hline Você confere os dados gerais do paciente? & Sim & 22 & $91,7 \%$ \\
& Às vezes & 2 & $8,3 \%$ \\
Você checa se o paciente realizou o jejum indicado? & Sim & 23 & $95,8 \%$ \\
& Às vezes & 1 & $4,2 \%$ \\
Você verifica antecedentes alérgicos do paciente? & Sim & 24 & $100 \%$ \\
Você verifica se o paciente trouxe exames anteriores? & Sim & 21 & $87,5 \%$ \\
& Não & 1 & $4,2 \%$ \\
& Às vezes & 2 & $8,3 \%$ \\
\hline
\end{tabular}

Fonte: dados da pesquisa. 
As informações apresentadas na Tabela 3, identificação do paciente e o orientam a respeito a seguir, referem que todos os profissionais da do posicionamento cirúrgico. Em contraponto, 18 equipe de enfermagem afirmam que na instituição utiliza-se um protocolo e um checklist de cirurgia segura. Estes profissionais também confirmam a (75\%) dos integrantes da equipe atentam para a acessibilidade de exames de imagens necessários do paciente.

Tabela 3 - Avaliação da equipe de enfermagem da unidade de centro cirúrgico de uma instituição hospitalar privada da Serra Gaúcha, referente ao momento de entrada e permanência do paciente na sala cirúrgica, antes da indução anestésica. 2020.

\begin{tabular}{lccc}
\hline Variáveis & Alternativas & $\mathbf{N}$ & $\mathbf{\%}$ \\
\hline Na instituição utiliza-se checklist nas cirurgias? & Sim & 24 & $100 \%$ \\
Na instituição utiliza-se algum protocolo de cirurgia segura? & Sim & 24 & $100 \%$ \\
A equipe de enfermagem confirma a identificação do paciente? & Sim & 24 & $100 \%$ \\
A equipe de enfermagem confirma qual procedimento o paciente & Sim & 23 & $95,8 \%$ \\
irá realizar? & Às vezes & 1 & $4,2 \%$ \\
A equipe de enfermagem confirma visualmente o sítio cirúrgico correto & Sim & 23 & $95,8 \%$ \\
e a sua demarcação? & Às vezes & 1 & $4,2 \%$ \\
A equipe de enfermagem confere a conexão do monitor multiparâmetro & Sim & 21 & $87 \%$ \\
ao paciente e seu funcionamento? & Não & 3 & $13 \%$ \\
A equipe de enfermagem atenta-se junto do anestesista e cirurgião para & Sim & 21 & $87,5 \%$ \\
eventos críticos previstos (perda sanguínea; via aérea de difícil acesso)? & Não & 1 & $4,2 \%$ \\
& Às vezes & 2 & $8,3 \%$ \\
\hline
\end{tabular}

Fonte: dados da pesquisa.

Na Tabela 4, conforme é possível visualizar nas informações apresentadas, 17 (70,8\%) dos profissionais realizam a contagem de instrumentos, compressas e agulhas antes do paciente sair da sala cirúrgica. No que diz respeito ao pós-operatório, $15(62,5 \%)$ revisam o plano de cuidados com o cirurgião. E, ainda, 22 (91,7\%) alegam documentar possíveis problemas com equipamentos.

Tabela 4 - Avaliação da equipe de enfermagem da unidade de centro cirúrgico de uma instituição hospitalar privada da Serra Gaúcha, referente ao momento antes do paciente sair da sala de cirurgia. 2020.

\begin{tabular}{lccc}
\hline Variáveis & Alternativas & N & \% \\
\hline Quando está circulando ou instrumentando você realiza a contagem & Sim & 17 & $70,8 \%$ \\
de instrumentos, compressas e agulhas? & Não & 3 & $12,5 \%$ \\
& Às vezes & 4 & $16,7 \%$ \\
Você documenta problemas com equipamentos? & Sim & 22 & $91,7 \%$ \\
& Não & 1 & $4,15 \%$ \\
& Às vezes & 1 & $4,15 \%$ \\
\hline
\end{tabular}

Fonte: dados da pesquisa. 
De acordo com os dados informados na Tabela 5, 12 (54,2\%) dos profissionais responderam que na instituição não há pessoal o suficiente para dar conta da carga de trabalho. Em contrapartida, $11(45,8 \%)$ alegam que na unidade há pessoal o suficiente. Quanto às horas de trabalho, 12 (54\%) afirmam que, às vezes, na unidade trabalha-se mais horas do que seria o melhor para o cuidado com o paciente. Ainda, 15 (62,5\%) negam que na unidade tem-se problema de segurança com o paciente.

Tabela 5 - Avaliação da equipe de enfermagem da unidade de centro cirúrgico de uma instituição hospitalar privada da Serra Gaúcha, referente à instituição e à unidade. 2020.

\begin{tabular}{lccc}
\hline Variáveis & Alternativas & N & \% \\
\hline Nesta unidade há pessoal o suficiente para executar a carga de trabalho? & Sim & 11 & $45,8 \%$ \\
& Não & 13 & $54,2 \%$ \\
Os profissionais de enfermagem desta unidade trabalham mais horas & Sim & 8 & $13 \%$ \\
do que seria o melhor para o cuidado do paciente? & Não & 3 & $33 \%$ \\
& Às vezes & 13 & $54 \%$ \\
Nesta unidade têm-se problemas de segurança com o paciente? & Sim & 2 & $8,3 \%$ \\
& Não & 15 & $62,5 \%$ \\
Nesta unidade os profissionais da enfermagem tomam atitudes & Às vezes & 7 & $29,2 \%$ \\
para melhorar a segurança do paciente? & Sim & 23 & $95,8 \%$ \\
O supervisor/chefe dá atenção suficiente aos problemas de segurança & Não & 1 & $4,2 \%$ \\
do paciente que acontecem repetidamente? & Sim & 23 & $95,8 \%$ \\
Nesta unidade debatem-se meios de prevenir erros? & Às vezes & 1 & $4,2 \%$ \\
A direção do hospital propicia um clima de trabalho que promova & Sim & 20 & $83,3 \%$ \\
a segurança do paciente? & Às vezes & 4 & $16,7 \%$ \\
\hline
\end{tabular}

Fonte: dados da pesquisa.

\section{Discussão}

Evidenciou-se que a maioria dos profissionais participantes do estudo era do sexo feminino $(91,7 \%)$, o que está alinhado com estudo de outros autores, que mencionam que a enfermagem traz o estereótipo de gênero, uma vez que se caracteriza por ser uma profissão predominantemente exercida e representada por mulheres. ${ }^{(9)}$ A média de idade dos participantes (31 a 40 anos) e o tempo de formação profissional predominante (6 anos a 10 anos) estão em concordância com outro estudo, que possui também uma mostra com idades entre 31 a 40 anos e com mais de 10 anos de formação.
Estes dados indicam que os profissionais participantes são enfermeiros e técnicos em enfermagem maduros, experientes, qualificados para o trabalho e que já desenvolveram certas habilidades e competências técnico-científicas que os permitem lidar com pessoas e situações complexas. ${ }^{(11)}$

Observou-se que todos os profissionais do estudo verificam os antecedentes alérgicos dos pacientes. Estima-se que as reações anafiláticas a anestésicos ocorram em 1:10.000-1:20.000 casos. A administração segura de medicamentos consiste na administração da droga correta ao paciente correto, na dose correta, no tempo correto, e pela via correta. Estudos avaliando erros de medicação 
demonstram que a equipe de saúde frequentemente falha em realizar estas etapas. Além da prática cuidadosa e atenção conscienciosa aos detalhes, é necessária uma abordagem baseada em sistemas para os processos de administração de drogas. ${ }^{(12)}$ Estudos realizados em Pernambuco, Brasil, reforçam a importância da checagem de antecedentes alérgicos no centro cirúrgico, pois neste local expõem-se os pacientes a diferentes classes medicamentosas, e as reações de anafilaxia podem ser ocasionadas por antibióticos, látex, bloqueadores neuromusculares, anestésicos locais, entre outros. Destarte, na presença de um agente desencadeador, o mesmo já deve ser substituído, prevenindo assim reações desnecessárias. ${ }^{(13)}$

Salienta-se que existem importantes estratégias para tornar o procedimento cirúrgico mais seguro e auxiliar a equipe de enfermagem a reduzir a possibilidade de ocorrência de danos ao paciente. Uma destas estratégias é o Protocolo para Cirurgia Segura, que tem como finalidade determinar as medidas a serem implantadas para reduzir a ocorrência de incidentes, eventos adversos e mortalidade cirúrgica, possibilitando o aumento da segurança na realização de procedimentos cirúrgicos, no local correto e no paciente correto, por meio do uso da Lista de Verificação de Cirurgia Segura (LVCS), também conhecida como checklist, desenvolvida pela Organização Mundial da Saúde (OMS). ${ }^{(8)}$ Nesse sentido, notou-se que todos os profissionais da equipe de enfermagem que participaram do estudo afirmam que na instituição utiliza-se um checklist e um protocolo de cirurgia segura.

Outra importante estratégia que tem por finalidade tornar o procedimento cirúrgico mais seguro é o programa Cirurgias Seguras Salvam Vidas, também criado pela OMS no ano de 2004. Este visa a redução da morbimortalidade de pacientes cirúrgicos, complementando as equipes cirúrgicas e os administradores hospitalares com orientações sobre a função de cada colaborador, que contempla dez objetivos essenciais para garantir a segurança do paciente. Este conjunto de objetivos pode ser considerado uma ferramenta para a segurança na prática dos profissionais de saúde, auxiliando-os no desenvolvimento de ações que visam a diminuição de erros nos processos assistenciais. ${ }^{(9)}$

Percebeu-se que todos os profissionais confirmam a identificação do paciente antes do inicio da cirurgia e $23(95,8 \%)$ confirmam qual procedimento o mesmo irá realizar. Assim, salienta-se a importância do Time Out, a fase de confirmação antes de ser realizada a incisão cutânea no paciente. Confere-se nessa etapa, verbalmente, pela equipe, os dados de identificação do paciente; assim, é mais uma oportunidade para a correção de algum fator não detectado ou não checado por parte de algum membro da equipe. ${ }^{(13)}$ Seu objetivo é assegurar que seja realizado o procedimento correto, no paciente correto e no local correto, de modo a proporcionar um cuidado seguro e de alta qualidade, permitindo que todas as questões não respondidas ou confusas sejam esclarecidas. Este consiste em uma prática baseada em evidências e opiniões de especialistas, que permite que a equipe cirúrgica possa seguir alguns passos de segurança e, assim, minimizar a ocorrência de eventos adversos para paciente. ${ }^{(14)}$

Ainda, antes da incisão cirúrgica, a equipe deve discutir o risco de grandes volumes de perdas sanguíneas e, se elas forem significativas, assegurar que o acesso endovenoso apropriado seja estabelecido. Na interpretação destes resultados, ressaltase positivamente os dados encontrados, pois $87,5 \%$ dos participantes atentam-se para eventos críticos previstos, como é o caso da perda sanguínea. A equipe envolvida em uma cirurgia deve estar atenta sobre o potencial de perda sanguínea importante antes do procedimento, além de preparar-se para isso. ${ }^{(11)}$ Deve-se prever reposição de fluídos e reservas de hemocomponentes, conforme orientações do protocolo nacional de cirurgia segura. Destaca-se que a transfusão é importante quando se tem o objetivo de manter os níveis de fatores de coagulação, hemoglobina e volume sanguíneo para a segurança durante o procedimento. Evita-se, com isso, por exemplo, o choque hipovolêmico. ${ }^{(13)}$

Ressalta-se que, de acordo com a OMS em publicação do ano de 2004, o objetivo para uma cirurgia segura é garantir que o procedimento seja realizado conforme planejado, atendendo aos 
"5 Certos": paciente (deve-se instituir e assegurar a Política ou Programa de Identificação do paciente); procedimento (deve-se instituir e assegurar a implementação de protocolos, embasamentos científicos e boas práticas); lateralidade (deve-se instituir e assegurar a prática internacional destinada a cirurgias e outros procedimentos invasivos em que haja a necessidade da escolha de um lado do corpo); posicionamento (deve-se seguir metodologias de posicionamento embasadas de acordo com cada tipo de cirurgia); e equipamentos (devese assegurar que existe um estoque mínimo de equipamentos, materiais e insumos). ${ }^{(8)}$

Constatou-se que a maioria dos profissionais atenta-se para a conexão do monitor multiparâmetro ao paciente, o que corrobora outro estudo realizado no Brasil - um resultado positivo de 93,7\%. Recomenda-se cumprir normas de monitorização mínima, independentemente do tipo de anestésico, duração deste e local. Torna-se imprescindível para o paciente cirúrgico ter disponíveis equipamentos de monitoramento, como um instrumento para a aferição de pressão arterial indireta e oxímetro de pulso. $^{(13)}$

Ademais, torna-se pertinente ressaltar que o posicionamento cirúrgico é um dos grandes fatores que colocam a integridade do paciente em risco durante o período transoperatório. Isso ocorre pois o paciente fica exposto e vulnerável por horas. Por conta disso, o enfermeiro tem como uma de suas funções a participação ativa no posicionamento do paciente em sala cirúrgica. Recai sobre este profissional a responsabilidade de planejar e implementar medidas e cuidados, que possibilitem a prevenção de lesões e de queimaduras, que podem ocorrer. ${ }^{(14)}$ Em um estudo realizado no Brasil, refere-se que a maioria dos pacientes apresentou alto risco para lesões perioperatórias, com média de 19,97.(15)

Considera-se o centro cirúrgico como um local onde muitas vezes há dificuldade de intubar pacientes ou manter sua ventilação manual sob máscara, e tal setor deve agrupar material diferenciado para casos previsíveis e não previsíveis, como laringoscópios com lâminas especiais. ${ }^{(13)} \mathrm{A}$ segurança das vias aéreas de um paciente submetido à anestesia geral é o evento isolado mais crítico durante a indução anestésica. No passado, os resultados adversos associados a eventos respiratórios foram os maiores tipos de injúria no Projeto Closed Claims ("acionamentos fechados") da Sociedade Americana de Anestesiologistas (ASA). O manejo inadequado de uma via aérea bloqueada, além da identificação inadequada do seu risco, continua a contribuir para a prevenção da mortalidade associada à anestesiologia em todo o mundo. ${ }^{(12)}$

O presente estudo mostrou que $70,8 \%$ da equipe de enfermagem participante realizam a contagem de instrumentos, compressas e agulhas. Esquecer uma compressa, agulha ou instrumental inadvertidamente em um paciente ao final de uma operação é um erro cirúrgico considerado raro, porém sério e persistente. Compressas e instrumentais retidos tendem a resultar em sérias sequelas, incluindo infecção, reoperação para remoção, perfuração intestinal, fístula ou obstrução e até mesmo óbito. Vários fatores contribuem para este erro, mas as evidências apontam para três fatores de risco claros: cirurgia de emergência, alto índice de massa corpórea e uma mudança não planejada na cirurgia. ${ }^{(12)} \mathrm{A}$ entrada e saída de todos os itens do campo operatório devem ser documentadas, evitando a ocorrência de erros de retenção. A literatura nacional preconiza a padronização do processo de contagem, pois essa medida pode auxiliar na prevenção de retenção de itens cirúrgicos. ${ }^{(16)}$

Ainda, recomenda-se ao final da cirurgia checar se algum aparelho apresentou problemas, para serem resolvidos antes da próxima intervenção. Com a checagem de aparelhos, será promovida a antecipação de eventuais problemas. ${ }^{(13)}$ Neste estudo isto evidenciou-se positivamente, uma vez que $91,7 \%$ dos profissionais realizam esta função. Uma situação frequente na estrutura organizacional da instituição hospitalar em geral é o quantitativo dos profissionais de enfermagem, geralmente em número insuficiente em relação à demanda das necessidades de um processo de cuidar integral e humanizado, conforme confirmou-se no presente estudo, onde 13 (54,2\%) dos participantes referem que na unidade não há funcionários 
suficientes para conseguir executar a carga de trabalho. De acordo com alguns autores, este aspecto pode resultar em uma priorização das atividades gerenciais e dos cuidados instrumentais em relação aos do tipo expressivo, determinante para a construção de uma base sustentadora no cuidado junto ao paciente. Além do mais, isto acaba também por afetar o ambiente psicológico dos profissionais que se sentem pressionados e assoberbados com suas inúmeras atribuições, que recai diretamente sobre o desenvolvimento de suas ações, prejudicando, por vezes, o diálogo, a troca de informações e experiências, e o desenvolvimento de um trabalho harmônico e solidário, fragilizando as relações estabelecidas entre eles. ${ }^{(17)}$

Além disso, estudos realizados no Rio de Janeiro, Brasil, apontam a importância de um ambiente humanizado que inclui uma assistência também humanizada, em que se busque não somente o bem-estar do paciente, mas também dos profissionais de saúde que nele atuam. ${ }^{(17)}$ Complementa-se que fatores, tais como a sobrecarga da jornada de trabalho, equipamentos e materiais com defeitos, o acúmulo de muitas atividades, e o número de profissionais insuficientes para a escala por motivos de férias, licenças ou outros afastamentos aumentam a fadiga e o estresse, dificultando a realização de ações que promovam a segurança do paciente, pois contribuem para o desgaste dos profissionais, prejudicando, por sua vez, a atenção durante a realização de procedimentos, e limitando sua habilidade de raciocinar. ${ }^{(18)}$

\section{Conclusão}

Constatou-se predomínio de concordância entre os enfermeiros e os técnicos em enfermagem em relação à adesão da segurança do paciente em centro cirúrgico. Todavia, a pesquisa também ressaltou as dificuldades que os profissionais relatam durante sua jornada de trabalho. Ainda, torna-se evidente a insatisfação dos mesmos em relação ao quantitativo dos profissionais de enfermagem referente à demanda das necessidades de um processo de cuidar e de segurança do paciente.
Para se realizar a prática de segurança do paciente, com qualidade, em uma unidade de alta complexidade, como é caso do centro cirúrgico, é notório que, além dos conhecimentos da equipe e dos protocolos da instituição para realizar uma prática segura, também é necessária a reestruturação do serviço para que os mesmos sejam capazes de desenvolver suas atribuições.

Sendo assim, os resultados desta pesquisa poderão auxiliar a prática de gestores e profissionais da enfermagem no desenvolvimento de estratégias para promoção da segurança do paciente no centro cirúrgico.

Quanto às limitações do estudo, notou-se que a amostragem foi menor que se esperava. Isto pode ter ocorrido devido à pandemia de Covid-19, pois não foi possível realizar a coleta de dados conforme o plano descrito inicialmente no projeto de pesquisa.

\section{Referências}

1 Henriques AHB, COSTA SS, Lacerda JS. Nursing care in surgical patient safety: an integrative review. Cogitare Enferm. [Internet]. 2016 [cited 2021 jul 15]; 21(4):01-08. Available from: https://revistas.ufpr.br/cogitare/article/ view/45622

2 Lopes TMR, Machado AVA, Silva AS, Santos TJX, Raiol IF, Miranda SA, et al. Atuação do enfermeiro na segurança do paciente em centro cirúrgico: revisão integrativa da literatura. REAS/ EJCHR. [Internet]. 2019 [citado 2021 jul 12]; (26):e769. Disponível em: https://acervomais. com.br/index.php/saude/article/view/769

3 Gutierres LS, Santos JLG, Peiter CC, Menegon FHA, Sebold LF, Erdmann AL. Good practices for patient safety in the operating room: nurses' recommendations. Rev Bras Enferm. [Internet]. 2018 [cited 2021 oct 12]; 71(Suppl 6):2775-82. Avaliable from: doi: http://dx.doi. org/10.1590/0034-7167-2018-0449

4 Cavalcante AKCB, Rocha RC, Nogueira LT, Avelino FVSD, Rocha SS. Cuidado seguro ao paciente: contribuições da enfermagem. Rev Cub 
Enferm. [Internet]. 2015 [citado 2021 out 21]; 31(4). Disponible en: http://www. revenfermeria.sld.cu/index.php/enf/article/ view/907

5 Agência Nacional de Vigilância Sanitária (BR). Protocolo para cirurgia segura. [Internet]. Rio de Janeiro: Fiocruz; 2013 [citado 2021 out 11]. Disponível em: https://www20.anvisa.gov.br/ segurancadopaciente/index.php/publicacoes/ item/protocolo-de-cirurgia-segura

6 Reis CT. A cultura de segurança do paciente: validação de um instrumento de mensuração para o contexto hospitalar brasileiro. [Internet]. [tese]. Rio de Janeiro: Escola Nacional de Saúde Pública Sergio Arouca; 2013 [citado 2021 out 11]. Disponível em: https://www. arca.fiocruz.br/handle/icict/14358

7 Ministério da Saúde (BR). Conselho Nacional de Saúde (CNS). Resolução no 466, de 12 de dezembro de 2012. [Internet]. Brasília; 2012 [citado 2021 out 13]. Disponível em: https:// bvsms.saude.gov.br/bvs/saudelegis/cns/2013/ res0466_12_12_2012.html

8 Ribeiro WA, Mattos IF, Morais MC, Souza DMS, Couto CS, Martins LM. Cirurgia segura: a enfermagem protagonizando a segurança do paciente no Centro Cirúrgico. Rev Pró-Univer SUS. [Internet]. 2019 [citado 2021 out 14]; 10(01):66-71. Disponível em: http://editora. universidadedevassouras.edu.br/index.php/ RPU/article/view/1684

9 Botelho ARM, Soares CC, Rodrigues EQ, Santos ELF, Santos RM, Costa CCP, et al. A atuação do enfermeiro na segurança do paciente em centro cirúrgico de acordo com os protocolos de cirurgia segura e segurança do paciente. Rev Presença. [Internet]. 2018 [citado 2021 out 12]; 4(10):1-28. Disponível em: http://revistapresenca.celsolisboa.edu.br/ index.php/numerohum/article/view/138

10 Cunha YFF, Sousa RR. Gênero e enfermagem: um ensaio sobre a inserção do homem no exercício da enfermagem. RAHIS. [Internet]. 2016 [citado 2021 out 14]; 13(3):140-49. Disponível em: https://revistas.face.ufmg.br/index.php/ rahis/article/view/140-149
11 Ribas EN. Enfermeira de ligação: estratégia de integração entre hospital e unidades da atenção primária. [Internet]. [dissertação]. Curitiba: Universidade Federal do Paraná; 2016 [citado 2021 maio 3]. Disponível em: https://www. scielo.br/j/reben/a/nQPG7QDTLGGrfnMWp wyPymk/?format=pdf HYPERLINK

12 Organização Mundial da Saúde. Segundo desafio global para a segurança do paciente: cirurgias seguras salvam vidas (orientações para cirurgia segura da OMS). [Internet]. Nilo MS; Durán IA, tradutores. Rio de Janeiro: Organização Pan-Americana da Saúde; 2009 [citado 2021 out 11]. Disponível em: http://bvsms. saude.gov.br/bvs/publicacoes/seguranca paciente_cirurgias_seguras_salvam_vidas

13 Pereira LFML, Oliveira SAR, Gomes GG. Segurança do paciente no transoperatório: análise do protocolo de cirurgia segura. Rev Enferm UFPE online. [Internet]. 2020 [citado 2021 set 14]; (14)e242554. Disponível em: https://periodicos.ufpe.br/revistas/ revistaenfermagem/article/download/ $242554 / 34346$

14 Martins GS, Carvalho R. Realização do timeout pela equipe cirúrgica: facilidades e dificuldades. Rev SOBECC. [Internet]. 2014 [citado 2021 out 15]; 19(1): Disponível em: http://doi. editoracubo.com.br/10.4322/sobecc.2014.007

15 Nascimento FCL, Rodrigues MCS. Risco de lesão por posicionamento cirúrgico: validação de escala em um hospital de reabilitação. Rev Latino-Am Enferm. [Internet]. 2020 [citado 2021 out 13]; (28) e3261. Disponível em: https://doi.org/10.1590/1518-8345.2912.3261

16 Freitas PS, Mendes KDS, Galvão CM. Processo de contagem cirúrgica: evidências para a segurança do paciente. Rev Gaúcha Enferm. [Internet]. 2016 [citado 2021 out 11]; 4:e66877. Disponível em: https://www.scielo. br/j/rgenf/a/r3t9YfDf5vSzD4PDNmMBHHL/ ?lang $=\mathrm{pt}$

17 Silva DC, Alvim NAT. Ambiente do Centro Ambiente do Centro Cirúrgico e os elementos que o integram: implicações para os cuidados de enfermagem. Rev Bras Enferm. [Internet]. 
2010 [citado 2021 out 12]. Disponível em: http://nascecme.com.br/2014/wp-content/ uploads/2015/01/centrocirurgico

18 Barradas NNUF, Guimarães DBO, Mendes PM, Abreu IM, Avelino FVSD, Dias SRS, et al. Atitudes de segurança da equipe de enfermagem no ambiente hospitalar. Rev Enferm UFPE online. 2019; 13:e239908. Disponível em: https://doi.org/10.5205/1981-8963.2019. 239908 
Ribeiro, B.; Souza, J. S. M. 